\title{
Two-sided Bar Constructions for Partial Monoids and Applications to $\boldsymbol{K}$-Homology Theory
}

Dai Tamaki

Department of Mathematical Sciences, Shinshu University, Matsumoto, 390-8621, Japan

rivulus@math.shinshu-u.ac.jp

\section{Introduction}

The aim of this article is to provide a method for proving a homotopy functor to be linear. The main result of this article is used in Tam to prove the Segal's $K$-homology and its twisted version are linear functors.

Theorem 1. Let $M$ be a partial topological monoid with a good unit acting on $X$ and $Y$ from the right and the left, respectively. If the inclusions

$$
\begin{aligned}
B_{n}(X, M, Y) & \hookrightarrow X \times M^{n} \times Y \\
B_{n}(X, M, *) & \hookrightarrow X \times M^{n} \\
C_{M}(Y) & \hookrightarrow M \times Y
\end{aligned}
$$

are weak equivalences for each $n$ and if the action of $m \in M$ on $Y$ induces a weak equivalence

$$
Y_{m}=\left\{y \in Y \mid(m, y) \in C_{M}(Y)\right\} \stackrel{m \cdot}{\longrightarrow} Y
$$

for each $m \in M$, then

$$
p^{Y}:\left|B_{*}(X, M, Y)\right| \longrightarrow\left|B_{*}(X, M, *)\right|
$$

is a quasifibration.

We remark that Shimakawa studied linear functors arising from partial Abelian monoids in Shi01, Shi07. The functors studied by Shimakawa overlap untwisted cases of functors studied in this paper, but he proved linearities by using a result of Woolfson Woo79 instead of the bar constructions on partial topological monoids. Although Shimakawa's and our results are based on a common idea of Segal's, our approach is more straightforward. 
As examples, we verify functors constructed from configuration spaces and Madsen-Tillmann spectrum [MT01] satisfy the conditions of the above theorem, hence are linear.

The paper is organized as follows:

1. In $₫ 2$, we use May's generalization of the bar construction and prove Theorem 1 .

2. In $\$ 3$, we explore a couple of examples and pose some questions.

We use notations and definitions introduced in a separate paper Tam freely.

Acknowledgement. The main theorem in this paper was proved when the author was preparing for a talk in a seminar at Kinosaki in 2005. The author is grateful to organizers of the Kinosaki meeting, especially D. Kishimoto, S. Kaji, T. Matsuoka, and A. Kono for inviting him to give a talk. The author would also like to express his sincere gratitude to the organizers of the conference on $D$-branes and $K$-theory held at Shonan in 2008 for creating an excellent environment, where the author explored a twisted version of Segal's $K$-homology theory.

This work is partially supported by Grants-in-Aid for Scientific Research, Ministry of Education, Culture, Sports, Science and Technology, Japan: 17540070.

\section{The Bar Construction on Monad}

As we have observed in Tam, a classical theorem of Dold and Thom DT58] can be interpreted as follows: the infinite symmetric product functor $\mathrm{SP}^{\infty}$ converts a diagram of based maps

$$
X \stackrel{f}{\longleftarrow} Y \stackrel{g}{\longrightarrow} Z
$$

into a quasifibration

$$
\mathrm{SP}^{\infty}(Z) \longrightarrow \mathrm{SP}^{\infty}\left(M_{f, g}\right) \longrightarrow \mathrm{SP}^{\infty}\left(X \cup_{f} C Y\right)
$$

It is also noticed in Tam that there is a functorial homeomorphism

$$
\mathrm{SP}^{\infty}\left(M_{f, g}\right) \cong\left|B_{*}\left(\mathrm{SP}^{\infty}(Z), \mathrm{SP}^{\infty}(X), \mathrm{SP}^{\infty}(Y)\right)\right|,
$$

where $B_{*}(-,-,-)$ is the two-sided geometric bar construction and $|-|$ is the geometric realization functor on simplicial spaces. The above quasifibration is obtained from a sequence of simplicial spaces

$$
\mathrm{SP}^{\infty}(Z) \longrightarrow B_{*}\left(\mathrm{SP}^{\infty}(Z), \mathrm{SP}^{\infty}(X), \mathrm{SP}^{\infty}(Y)\right) \longrightarrow B_{*}\left(*, \mathrm{SP}^{\infty}(X), \mathrm{SP}^{\infty}(Y)\right)
$$

by taking the geometric realization functor. Thus the main theorem of Dold and Thom is a consequence of the identification (11) and a general fact on the two-sided bar construction on topological monoid proved, for example, in Mil67, May75. 
Theorem 2. Let $G$ be a topological monoid acting on $X$ and $Y$ from the right and the left, respectively. If $(G,\{e\})$ is a strong $N D R$ pair and $\pi_{0}(G)$ is a group, then the following sequence is a quasifibration

$$
Y \longrightarrow\left|B_{*}(X, G, Y)\right| \stackrel{p}{\longrightarrow}\left|B_{*}(X, G, *)\right|,
$$

where $p$ is given by collapsing $Y$ to a single point.

The functor $\mathrm{SP}^{\infty}$ is exceptionally well-behaved. Although it is well-known Goo03 that any homology theory $\widetilde{E}_{*}(-)$ can be expressed as a composition of a space-level functor

$$
F_{E}: \operatorname{Spaces}_{*} \longrightarrow \text { Spaces }_{*}
$$

and the homotopy groups functor

$$
\widetilde{E}_{*}(X) \cong \pi_{*}\left(F_{E}(X)\right),
$$

it is rarely the case that $F_{E}$ converts double mapping cylinders to two-sided bar constructions.

For example, consider Segal's $K$-homology theory Seg77] $k u(-; H)$ based on a Hilbert space $H$. It is shown in Tam that $k u(-; H)$ converts double mapping cylinders to certain subspaces of the two-sided bar construction

$$
k u\left(M_{f, g} ; H\right) \cong\left|B_{*}^{\perp}(k u(Z ; H), k u(X ; H), k u(Y ; H))\right| .
$$

We can not apply the classical theory of two-sided bar constructions on topological monoids, although we have a sequence of simplicial spaces

$k u(Z ; H) \longrightarrow B_{*}^{\perp}(k u(Z ; H), k u(X ; H), k u(Y ; H)) \longrightarrow B_{*}^{\perp}(*, k u(X ; H), k u(Y ; H))$.

Thus it is natural to ask the following question.

Question 1. When does a map of simplicial spaces

$$
f_{*}: X_{*} \longrightarrow Y_{*}
$$

induces a quasifibration

$$
\left|f_{*}\right|:\left|X_{*}\right| \longrightarrow\left|Y_{*}\right|
$$

after taking the geometric realization?

J.P. May gives an answer to this question in May72 by introducing the notion of simplicial Hurewicz fibration. However, the definition of simplicial Hurewicz fibration is complicated and it is not easy to use May's theorem for our purpose. Another condition was found by Anderson And78. His condition involves the fundamental groupoid of the base space.

Let us restrict our attention to the simplicial spaces which arise as "generalized bar constructions" and consider the above question. We would like to be a bit more general and introduce a construction for partial monoids, which is analogous to the two-sided bar construction for topological monoids. 
Definition 1. A partial (topological) monoid is a pointed topological space $(M, *)$ together with a subspace $C(M) \subset M \times M$ and a map

$$
\mu_{M}: C(M) \longrightarrow M
$$

satisfying the following conditions:

1. $\left(m_{1}, m_{2}\right) \in C(M)$ and $\left(\mu_{M}\left(m_{1}, m_{2}\right), m_{3}\right) \in C(M)$ if and only if $\left(m_{2}, m_{3}\right) \in$ $C(M)$ and $\left(m_{1}, \mu_{M}\left(m_{2}, m_{3}\right)\right) \in C(M)$. And

$$
\mu_{M}\left(\mu_{M}\left(m_{1}, m_{2}\right), m_{3}\right)=\mu_{M}\left(m_{1}, \mu_{M}\left(m_{2}, m_{3}\right)\right)
$$

for such a triple $\left.\left(m_{1}, m_{2}, m_{3}\right) \in\left(\mu_{M} \times 1\right)^{-1}(C(M))=1 \times \mu_{M}\right)^{-1}(C(M))$. 2. $M \vee M \subset C(M)$ and the following diagram is commutative

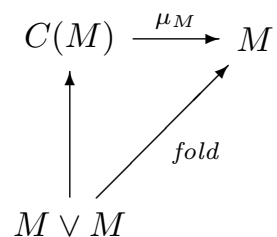

The set $C(M)$ is called the set of composable pairs.

Definition 2. Let $M$ be a partial monoid. A left action of $M$ on a space $X$ is map

$$
\mu_{X}: C_{M}(X) \longrightarrow X
$$

where $C_{M}(X) \subset M \times X$, satisfying the following conditions:

1. $\left(m_{2}, x\right) \in C_{M}(X)$ and $\left(m_{1}, \mu_{X}\left(m_{2}, x\right)\right) \in C_{M}(X)$ if and only if $\left(m_{1}, m_{2}\right) \in$ $C(M)$ and $\left(\mu_{M}\left(m_{1}, m_{2}\right), x\right) \in C_{M}(X)$. And

$$
\mu_{X}\left(m_{1}, \mu_{X}\left(m_{2}, x\right)\right)=\mu_{X}\left(\mu_{M}\left(m_{1}, m_{2}\right), x\right) .
$$

2. $\{*\} \times X \subset C_{M}(X)$ and the following diagram is commutative

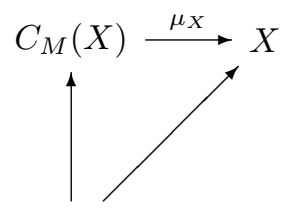

$\{*\} \times X$

$C_{M}(X)$ is also called the set of composable pairs.

$A$ right action is defined similarly. 
Example 1. For a pointed space $X$, Segal's construction $k u(X ; H)$ has a structure of partial monoid with

$$
C(k u(X ; H))=k u(X ; H) \stackrel{\perp}{\times} k u(X ; H) .
$$

The multiplication is given by the concatenation.

A based map

$$
f: X \longrightarrow Y
$$

induces a map of partial monoids

$$
f_{*}: k u(X ; H) \longrightarrow k u(Y ; H)
$$

hence an action of $k u(X ; H)$ on $k u(Y ; H)$ with the set of composable pairs

$$
C_{k u(X ; H)}(k u(Y ; H))=k u(X ; H) \stackrel{\perp}{\times} k u(Y ; H) .
$$

Analogously, the twisted version $k u^{A S}(\varphi, s ; H)$ constructed in Tam is a partial monoid and a morphism $f:(\varphi, s) \longrightarrow(\psi, t)$ induces an action of $k u^{A S}(\varphi, s)$ on $k u^{A S}(\psi, t)$.

A partial monoid naturally gives rise to a monad.

Definition 3. A monad in a category $\mathbf{C}$ is a covariant functor

$$
C: \mathbf{C} \longrightarrow \mathbf{C}
$$

together with natural transformations

$$
\begin{aligned}
& \mu: C^{2} \longrightarrow C \\
& \eta: 1 \longrightarrow C
\end{aligned}
$$

making the following diagrams commutative for each object $X$

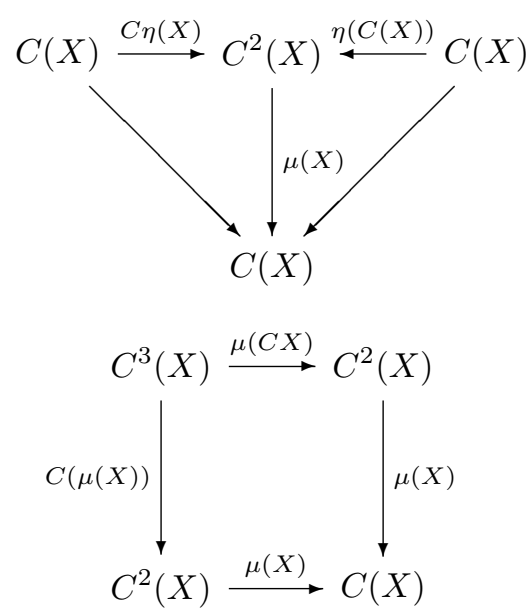


Definition 4. Let $C$ be a monad in a category C. An algebra over $C$ is an object $X$ in $\mathbf{C}$ together with a map

$$
\xi: C(X) \longrightarrow X
$$

making the following diagrams commutative

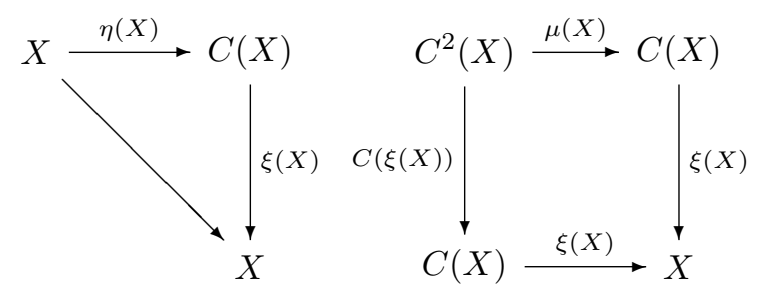

Example 2. Let $M$ be a partial monoid and $M$-Spaces be the category of left $M$-spaces. Then $C_{M}$ can be regarded as a monad in $M$-Spaces as follows. For an object $X$ in $M$-Spaces, we need to make $C_{M}(X)$ into an object of $M$-Spaces. The set of composable pairs is

$C_{M}\left(C_{M}(X)\right)=\left\{(m,(n, x)) \in M \times C_{M}(X) \mid(m, n) \in C(M),(m n, x) \in C_{M}(X)\right\}$

and the action of $M$ is the multiplication of $M$. Thus $C_{M}$ becomes an endofunctor

$$
C_{M}: M \text {-Spaces } \longrightarrow M \text {-Spaces. }
$$

It is obvious that $C_{M}$ is a monad in $M$-Spaces. In fact any object in $M$-Spaces is an algebra over $C_{M}$.

In order to extend the two-sided bar construction from monoids to monads, we also need the concept of a $C$-functor for a monad $C$.

Definition 5. Let $(C, \mu, \eta)$ be a monad in a category $\mathcal{C} . A$-functor in a category $\mathcal{D}$ is a functor

$$
F: \mathcal{C} \longrightarrow \mathcal{D}
$$

together with a natural transformation

$$
\lambda: F C \longrightarrow F
$$

making the following diagrams commutative
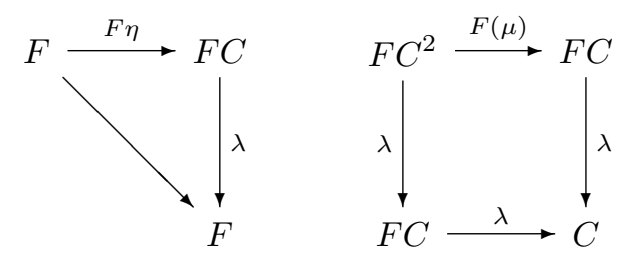
Example 3. Let $M$ be a partial monoid and $X$ be a right $M$-space. The functor

$$
C_{X}: M \text {-Spaces } \longrightarrow \text { Spaces }
$$

defined by

$$
C_{X}(Y)=\left\{\begin{array}{l|l}
(x, y) \in X \times Y & \begin{array}{l}
x=x^{\prime} m \Longrightarrow(m, y) \in C_{M}(Y) \\
y=m y^{\prime} \Longrightarrow(x, m) \in C_{M}(X)
\end{array}
\end{array}\right\}
$$

is a $C_{M}$-functor. The natural transformation

$$
\lambda: C_{X} C_{M} \longrightarrow C_{X}
$$

is given by the action of $M$ on $X$.

J.P. May introduced a generalization of the two-sided bar construction to the triple of a monad $C$, an algebra over $C$ and a $C$-functor.

Definition 6. Let $(C, \mu, \eta)$ be a monad in a category C. Given an algebra $(X, \xi)$ over $C$ and a $C$-functor $(F, \lambda)$ in $\mathbf{D}$, define a simplicial object $B_{*}(F, C, X)$ in $\mathbf{D}$ by

$$
B_{q}(F, C, X)=F C^{q}(X) .
$$

The face and degeneracy operators are given by

$$
\begin{aligned}
d_{0} & =\lambda \\
d_{i} & =F C^{i-1} \mu\left(C^{q-i}(X)\right) \text { for } 1 \leq i \leq q-1 \\
d_{q} & =F C^{q-1} \xi \\
s_{i} & =F C^{i} \eta\left(C^{q-i}(X)\right) .
\end{aligned}
$$

Consider a monad $C$ in a category $\mathbf{C}$ and a $C$-functor

$$
F: \mathbf{C} \longrightarrow \text { Spaces }
$$

For a map of $C$-algebras

$$
f: X \longrightarrow Y
$$

we obtain a map of simplicial spaces

$$
f_{*}: B_{*}(F, C, X) \longrightarrow B_{*}(F, C, Y) .
$$

In order to find a practical condition under which this map induces a quasifibration after taking the geometric realization, let us restrict our attention to the case of partial monoid.

Let $M$ be a partial monoid. Let $X$ and $Y$ be right and left $M$-spaces, respectively. The bar construction on $C_{M}, Y$, and $C_{X}$ is denoted by

$$
B_{*}(X, M, Y)=B_{*}\left(C_{X}, C_{M}, Y\right),
$$


for the sake of simplicity. We have the following commutative square of simplicial spaces

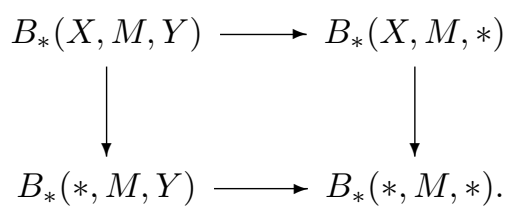

We would like to find a condition which makes the geometric realization of the above diagram homotopy Cartesian.

We use the following result of Segal $\operatorname{Seg} 74]$. Recall that $\Delta$ is the small category of finite totally order sets and order preserving maps so that a simplicial space $X_{*}$ is nothing but a functor

$$
X_{*}: \Delta^{\mathrm{op}} \longrightarrow \text { Spaces. }
$$

Definition 7. We say a simplicial space $X_{*}$ is strongly cofibrant if, for each face operator

$$
d_{i}: X_{n-1} \hookrightarrow X_{n},
$$

the pair $\left(X_{n}, d_{i}\left(X_{n-1}\right)\right)$ is a strong NDR pair.

Theorem 3. Let

$$
f_{*}: X_{*} \longrightarrow Y_{*}
$$

be a map of strongly cofibrant simplicial spaces satisfying the following condition: for each morphism $\theta: \boldsymbol{m} \longrightarrow \boldsymbol{n}$ in $\Delta$, the diagram

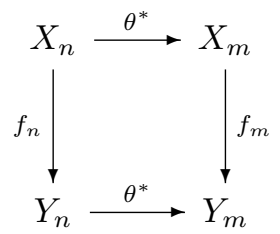

is homotopy Cartesian. Then the diagrams

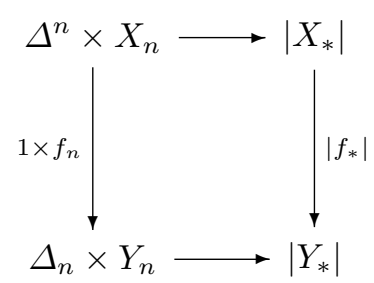

are homotopy Cartesian for all $n$.

In order to make use of this theorem, we need a condition under which strongly cofibrantness holds. The following is a reasonalbe condition having regard to Theorem 2 and follows immediately from the standard choice of NDR representation for a product of NDR pairs. 
Lemma 1. Let $(M, *)$ be a partial monoid. If $(M, *)$ has a strong NDR representation $(h, u)$ satisfying the condition that, if $\left(m_{1}, m_{2}\right) \in C(M)$ then $\left(h\left(m_{1}, s\right), h\left(m_{2}, t\right)\right) \in C(M)$ for all $s, t \in I$, then $B_{*}(X, M, Y)$ is a strongly cofibrant simplicial space for any right $M$-space $X$ and left $M$-space $Y$.

Proof. The standard NDR representation for the product of NDR pairs gives the desired strong NDR representation under the assumption of the Lemma.

We use the following terminology for simplicity.

Definition 8. We say a partial monoid $M$ has a good unit if $(M, *)$ has a strong NDR representation $(h, u)$ satisfying the condition that, if $\left(m_{1}, m_{2}\right) \in$ $C(M)$ then $\left(h\left(m_{1}, s\right), h\left(m_{2}, t\right)\right) \in C(M)$ for all $s, t \in I$.

The following is our answer to Question 1 in the case of the two-sided bar construction for partial monoid.

Theorem 4. Let $M$ be a partial monoid with a good unit acting on $X$ and $Y$ from the right and the left, respectively. If the inclusions

$$
\begin{aligned}
B_{n}(X, M, Y) & \hookrightarrow X \times M^{n} \times Y \\
B_{n}(X, M, *) & \hookrightarrow X \times M^{n} \\
C_{M}(Y) & \hookrightarrow M \times Y
\end{aligned}
$$

are weak homotopy equivalences for each $n$ and if the action of $m \in M$ on $Y$ induces a weak homotopy equivalence

$$
Y_{m}=\left\{y \in Y \mid(m, y) \in C_{M}(Y)\right\} \stackrel{m \cdot}{\longrightarrow} Y
$$

for each $m \in M$, then

$$
p^{Y}:\left|B_{*}(X, M, Y)\right| \longrightarrow\left|B_{*}(X, M, *)\right|
$$

is a quasifibration with fiber $Y$.

Proof. We are going to prove that the homotopy fiber of $p_{Y}$ over $[\boldsymbol{s}, \boldsymbol{x}] \in$ $\left|B_{*}(X, M, *)\right|$ is weakly homotopy equivalent to $Y$ under the canonical map.

Suppose

$$
p_{*}^{Y}: B_{*}(X, M, Y) \longrightarrow B_{*}(X, M, *),
$$

satisfies the condition for Theorem 3 , (We wiil check this later.) Take an element $[s, x] \in\left|B_{*}(X, M, *)\right|$ and consider the homotopy fiber over this element. This element is represented by an element $(s, x) \in \Delta^{n} \times B_{n}(X, M, *)$ for some $n$. By Theorem 3, we have the following homotopy Cartesian diagram

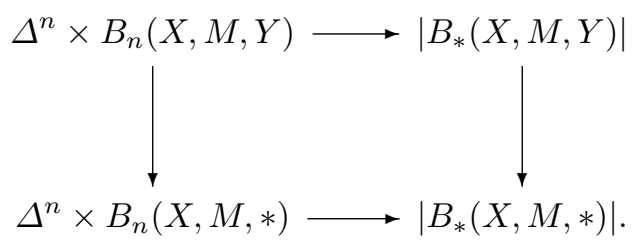


If we denote $F_{n}$ and $F$ for the homotopy fibers of

$$
\begin{gathered}
p_{n}^{Y}: B_{n}(X, M, Y) \longrightarrow B_{n}(X, M, *) \\
\left|p_{*}^{Y}\right|:\left|B_{*}(X, M, Y)\right| \longrightarrow\left|B_{*}(X, M, *)\right|,
\end{gathered}
$$

over $(s, x)$ and $[s, x]$, respectively, then it follows that

$$
F_{n} \stackrel{\stackrel{\widetilde{w}}{\longrightarrow}}{\longrightarrow} F
$$

In order to prove that $F_{n}$ is weakly equivalent to $Y$, let us extend the commutative diagram

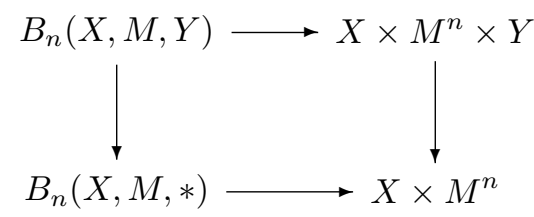

to the $3 \times 3$ diagram of homotopy fiber sequences. Then we have

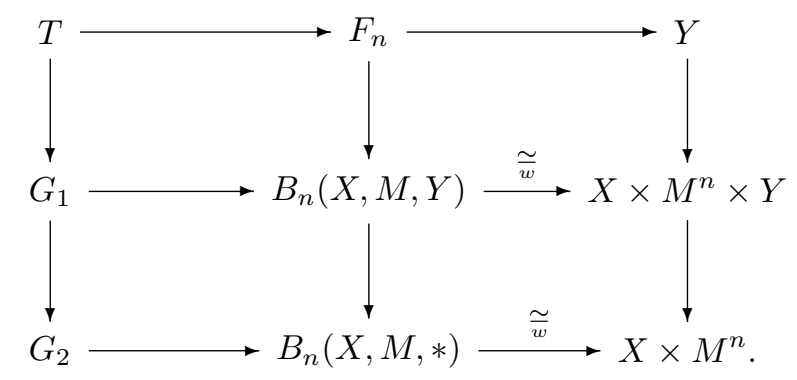

By assumption $G_{1}$ and $G_{2}$ are weakly contractible and hence $F_{n}$ and $Y$ are weakly equivalent.

Now it remains to check that our simplicial map $p_{*}^{Y}$ satisfies the condition for Theorem 3, It suffices to prove the following diagram is homotopy Cartesian for $\theta^{*}=d_{i}$ and $s_{i}$.

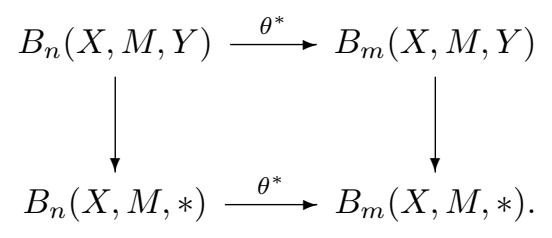

When $\theta^{*}=s_{i}$ for some $i$, we have the following commutative diagram 


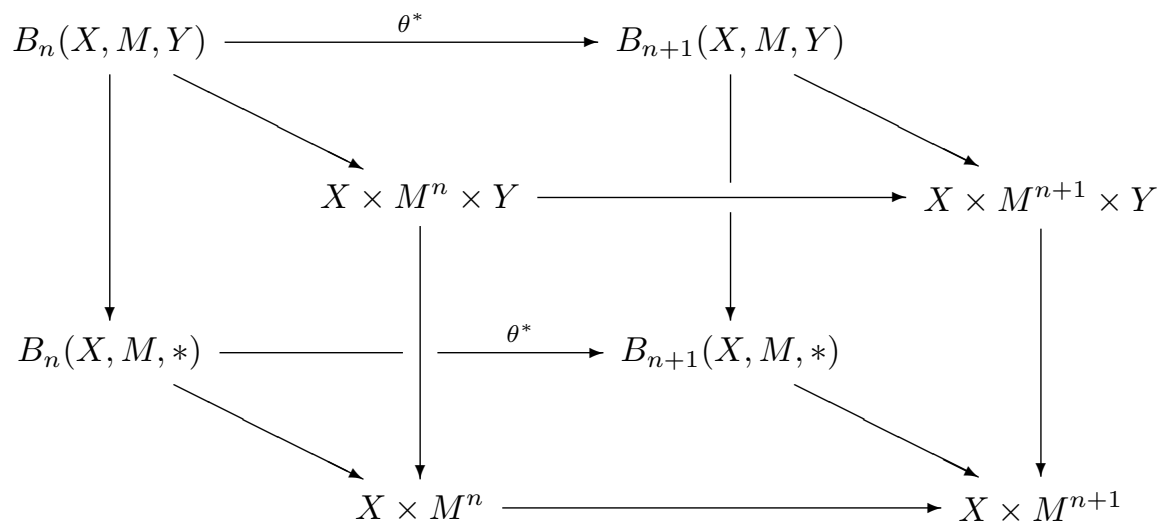

where the horizontal maps in the front diagram are given by inserting $*$ in $i$-th place of $M^{n+1}$.

For $d_{i}(0<i<n)$, we also have the following diagram

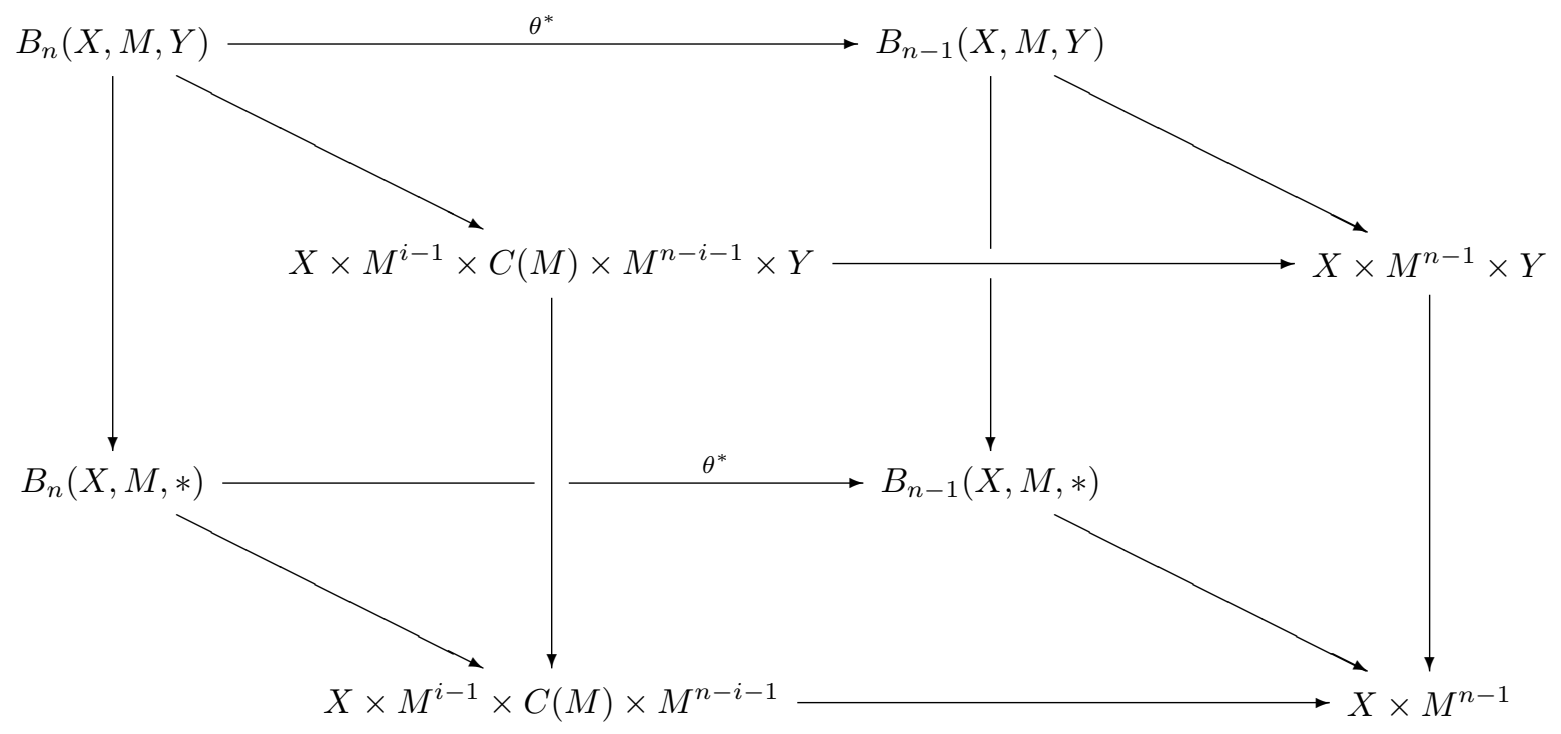

where the front horizontal maps are given by

$$
\mu_{M}: C(M) \longrightarrow M
$$

For $d_{0}$, we have 


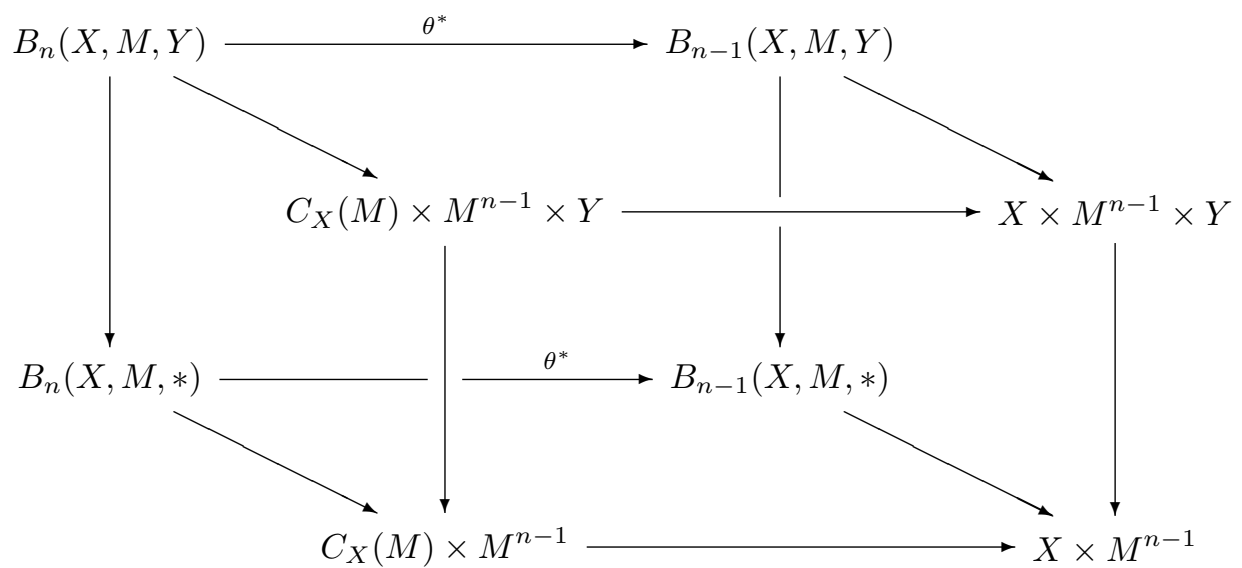

where the front horizontal maps are given by the action of $M$ on $X$.

In all these cases, the vertical maps in the front square of the diagrams are trivial fibrations with fiber $Y$ and the maps of diagrams from the back to the front are all weak equivalences. Thus the homotopy fibers of the vertical maps in the backside diagram are both weakly equivalent to $Y$ hence the backside diagrams are homotopy Cartesian.

The case of $d_{n}$ is a little bit different. We have the following diagram

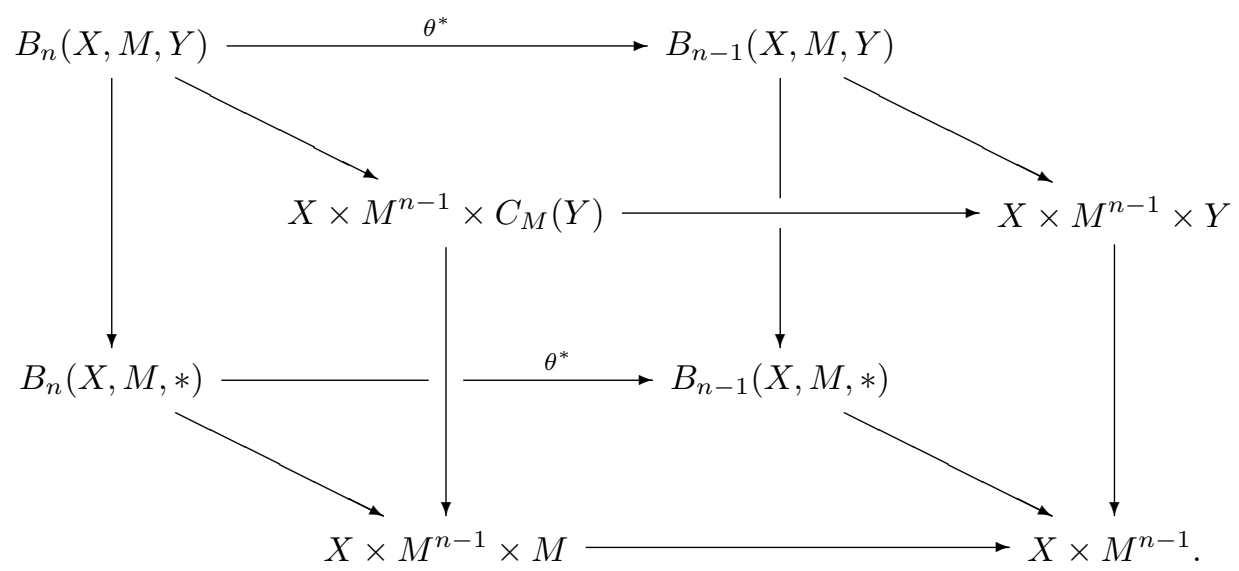

Thus it is enough to prove that the following diagram is homotopy Cartesian

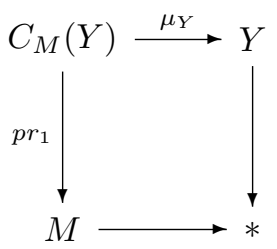

Note that, by assumption, $Y_{m}$ is weakly homotopy equivalent to the homotopy fiber of the projection 


$$
p r_{1}: C_{M}(Y) \longrightarrow Y \text {. }
$$

By extending this diagram, we obtain a $3 \times 3$ diagram of homotopy fiber sequences

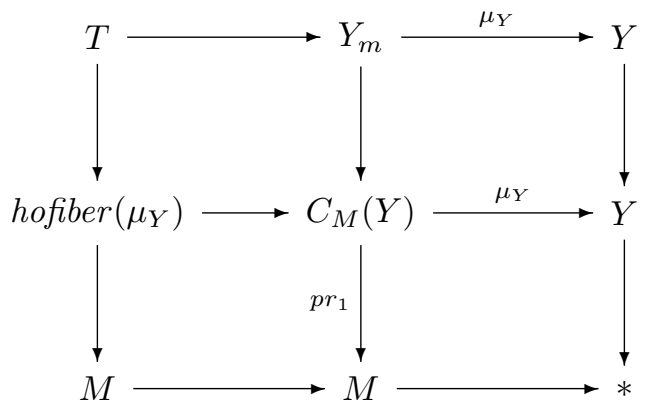

and the result follows from the assumption that $\left.\mu_{Y}\right|_{Y_{m}}$ is a weak equivalence.

We use Theorem 4 to prove the linearlity of the Segal's functor $k u(-; H)$ and its twisted version $k u^{A S}(-; H)$ in a separate paper Tam. Note that we can remove the path-connectedness condition by taking a homotopy theoretic group completion.

Corollary 1. The following functor is a reduced homology theory

$$
X \longmapsto \pi_{*}(\Omega k u(\Sigma X ; H)) .
$$

Corollary 2. The following functor is a reduced homology theory

$$
(\varphi, s) \longmapsto \pi_{*}\left(\Omega k u^{A S}\left(\Sigma_{B P U(H)}(\varphi, s ; H)\right)\right)
$$

on the category of ex-spaces over $B P U(H)$.

\section{Examples}

We have seen that the facts that the functors

$$
\begin{aligned}
X & \longmapsto \pi_{*}\left(\Omega \operatorname{SP}^{\infty}(\Sigma X)\right) \\
X & \longmapsto \pi_{*}(\Omega k u(\Sigma X ; H)) \\
(\varphi, s) & \longmapsto \pi_{*}\left(\Omega k u^{A S}(\varphi, s ; H)\right)
\end{aligned}
$$

define (reduced) homology theories follow immediately from Theorem 4 . Note that the dimension function

$$
\operatorname{dim}:\{\text { finite dimensional vector subspaces in } H\} \longrightarrow \mathbb{N} \cup\{0\}
$$

induces a canonical natural transformation of linear functors 


$$
\operatorname{dim}: k u(X ; H) \longrightarrow \mathrm{SP}^{\infty}(X),
$$

hence a natural transformation of homology theories.

Segal proves that $\pi_{*}(\Omega k o(\Sigma X))$ is the connective $K O$-theory by comparing $k o\left(S^{n}\right)$ with the Bott periodicity spaces. In this sense, the connective $K O$-homology is obtained by a categorification of $\mathbb{N} \cup\{0\}$. It seems that categorifications of $\mathbb{N} \cup\{0\}$, categorifications of categorifications of $\mathbb{N} \cup\{0\}$, and so on, give us interesting examples of homology theories.

Let us begin with the stable homotopy theory, whose construction in terms of configuration spaces have been investigated by many people [Seg73, Shi01].

Example 4. For a vector space $H$ over $\mathbb{R}$, let $\mathcal{C}(j ; H)$ be the space of (ordered) distinct $j$ points in $H$. For a pointed space $X$, define

$$
C(X ; H)=\left(\coprod_{j=0}^{\infty} \mathcal{C}(j ; H) \times_{\Sigma_{j}} X^{j}\right) / \sim
$$

where $\sim$ is the basepoint relation.

$C(X ; H)$ is a partial monoid under the concatenation: the set of composable pairs is

$$
C(C(X ; H))=\left\{([\boldsymbol{c}, \boldsymbol{x}]),\left(\left[\boldsymbol{c}^{\prime}, \boldsymbol{x}^{\prime}\right]\right) \in C(X ; H) \times C(X ; H) \mid \boldsymbol{c} \cap \boldsymbol{c}^{\prime}=\emptyset\right\} .
$$

When $H=\mathbb{R}^{\infty}, C(X ; H)$ satisfies the condition for Theorem 4 and it follows that

$$
X \longrightarrow \pi_{*}\left(\Omega C\left(\Sigma X ; \mathbb{R}^{\infty}\right)\right)
$$

is a homology theory. This is the stable homotopy group of $X, \pi_{*}^{S}(X)$.

Note that an element of $C\left(X ; \mathbb{R}^{\infty}\right)$ can be written as

$$
\left(\left[c_{1}, \cdots, c_{k} ; x_{1}, \cdots, x_{k}\right]\right)
$$

where $c_{i} \in \mathcal{C}\left(j_{i} ; \mathbb{R}^{\infty}\right)$ and $x_{i} \neq x_{i^{\prime}}$ if $i \neq i^{\prime}$. The cardinality function

$$
\coprod \mathcal{C}\left(j ; \mathbb{R}^{\infty}\right) \longrightarrow \mathbb{N}
$$

induces a natural transformation of homology theories

$$
\pi_{*}^{S}(X) \longrightarrow \widetilde{H}_{*}(X ; \mathbb{Z})
$$

which is nothing but the stable Hurewicz homomorphism.

We can also consider configuration spaces with multiplicity at most $i$ and obtain a homology theory $\pi_{*}\left(\Omega^{\infty} \mathrm{SP}^{i}\left(\Sigma^{\infty} X\right)\right)$ lying in between these two

$$
\pi_{*}^{S}(X) \longrightarrow \pi_{*}\left(\Omega^{\infty} \operatorname{SP}^{i}\left(\Sigma^{\infty} X\right)\right) \longrightarrow \widetilde{H}_{*}(X ; \mathbb{Z}) .
$$


Example 5. Consider the infinite loop space structure on

$$
\Omega B\left(\coprod_{g} B \Gamma_{g, 1}\right) \simeq \mathbb{Z} \times B \Gamma_{\infty}^{+}
$$

studied by Tillmann [Til97, MT01, where $\Gamma_{g, 1}$ is the mapping class group of oriented surface of genus $g$ with one boundary component,

$$
\Gamma_{\infty}=\operatorname{colim}_{g} \Gamma_{g, 1}
$$

by sewing a torus with two boundary components, and + in $B \Gamma_{\infty}^{+}$denotes the plus-construction of Quillen.

Following the construction of Madsen and Tilmann, we can form a topological category $\mathcal{Y}$ as follows: objects are given by

$$
\operatorname{Ob}(\mathcal{Y})=\coprod_{j=0}^{\infty} \operatorname{Emb}\left(S_{j}, \mathbb{R}^{\infty}\right) / \operatorname{Diff}\left(S_{j}\right)
$$

where $S_{j}=\underbrace{S^{1} \amalg \cdots \amalg S^{1}}_{j}$, Emb is the space of smooth embeddings, and Diff is the group of diffeomorphisms. The space of morphisms is given by cobordisms in $\mathbb{R}^{\infty}$. To be more precise, let $F$ be an oriented cobordism from $S_{n}$ to $S_{m}$, namely

$$
\begin{aligned}
& \partial_{-} F=S_{n}, \\
& \partial_{+} F=S_{m} .
\end{aligned}
$$

For $t>0$, let

$$
\operatorname{Emb}^{\Omega}\left(F ;[0, t] \times \mathbb{R}^{\infty}\right)
$$

be the space of embeddings $h F \hookrightarrow[0, t] \times \mathbb{R}^{\infty}$ with

$$
\begin{aligned}
& h\left(\partial_{-} F\right)=h(F) \cap\{0\} \times \mathbb{R}^{\infty} \\
& h\left(\partial_{+} F\right)=h(F) \cap\{t\} \times \mathbb{R}^{\infty} .
\end{aligned}
$$

We assume that the boundary of $F$ has good collar neighborhood and elements in $\operatorname{Emb}^{\Omega}\left(F ;[0, t] \times \mathbb{R}^{\infty}\right)$ map these collars into the good collars of $h(F)$. Let $\operatorname{Diff}^{\Omega}(F)$ be the group of diffeomorphisms of $F$ which restricts to give diffeomorphisms of the form

$$
\begin{aligned}
& \partial_{-} \phi \times 1_{[0, \varepsilon]}, \\
& \partial_{+} \phi \times 1_{[0, \varepsilon]}
\end{aligned}
$$

on the collar neighborhood of the incoming and outgoing boundaries. The space of morphisms in $\mathcal{Y}$ is given by 


$$
\operatorname{Mor}(\mathcal{Y})=\operatorname{Ob}(\mathcal{Y}) \amalg \coprod_{F, t>0} \operatorname{Emb}^{\Omega}\left(F,[0, t] \times \mathbb{R}^{\infty}\right) / \operatorname{Diff}^{\Omega}(F)
$$

with a suitable topology.

Note that the space of morphism of this category has two operations: disjoint union and sewing. Of course, the sewing operation is the composition of morphisms. The disjoint union can be taken only when the images are disjoint in $\mathbb{R}^{\infty}$. Thus the disjoint union operation gives a partial monoid structure on the nerve of $\mathcal{Y}$. In order to form a "balanced product" of $N_{*} \mathcal{Y}$ and a pointed space $X$ as we have done to define $k u(X ; H)$ or $C\left(X ; \mathbb{R}^{\infty}\right)$, we need to label the connected components of surfaces in the nerves of $\mathcal{Y}_{b}$. Thus we define a simplicial space $Y(j)_{*}$ by

$$
Y(j)_{q}=\left\{([\boldsymbol{h}], \lambda) \mid[\boldsymbol{h}] \in N_{q} \mathcal{Y}, \lambda: \pi_{0} \operatorname{Im}\left(h_{q} \circ \cdots \circ h_{1}\right) \rightarrow\{1, \cdots, j\}\right\} .
$$

For a pointed space $X$, define

$$
M_{q}(X)=\left(\coprod_{j=0}^{\infty} Y(j)_{q} \times_{\Sigma_{j}} X^{j}\right) / \sim
$$

where $\sim$ is the basepoint relation. Thus we have a simplicial space $M_{*}(X)$ with a partial monoid structure on each $M_{q}(X)$ defined by disjoint union. As in the case of Segal's $K$-homology, given pointed maps

$$
X \stackrel{f}{\longleftarrow} Y \stackrel{g}{\longrightarrow} Z
$$

we have a homeomorphism

$$
M_{q}\left(Y \cup_{f}(Y \times I) \cup_{g} Z\right) \cong\left|B_{*}\left(M_{q}(X), M_{q}(Y), M_{q}(Z)\right)\right| .
$$

Note that

$$
B_{*}\left(M_{*}(X), M_{*}(Y), M_{*}(Z)\right)=\left\{B_{p}\left(M_{q}(X), M_{q}(Y), M_{q}(Z)\right)\right\}_{p, q}
$$

is a bisimplicial space and by the standard property of bisimplicial space

$$
\begin{aligned}
\left|M_{*}\left(Y \cup_{f} Y \times I \cup_{g} Z\right)\right| & \cong\left(\coprod_{q \geq 0} \Delta^{q} \times\left|B_{*}\left(M_{q}(X), M_{q}(Y), M_{q}(Z)\right)\right|\right) / \sim \\
& \cong\left|B_{*}\left(\left|M_{*}(X)\right|,\left|M_{*}(Y)\right|,\left|M_{*}(Z)\right|\right)\right| .
\end{aligned}
$$

It is essentially proved by Madsen and Tillmann that the inclusion

$$
B_{q}\left(\left|M_{*}(X)\right|,\left|M_{*}(Y)\right|,\left|M_{*}(Z)\right|\right) \hookrightarrow\left|M_{*}(X)\right| \times\left|M_{*}(Y)\right|^{q} \times\left|M_{*}(Z)\right|
$$

is a homotopy equivalence (Theorem 2.3 of [MT01]). Thus we can apply Theorem 4 and 


$$
X \longrightarrow \pi_{*}\left(\Omega\left|M_{*}(\Sigma X)\right|\right)
$$

is a homology theory.

Note that this homology theory is larger than the stable homotopy theoy (away from 2) $\pi_{*}^{S}(X)_{\left[\frac{1}{2}\right]}$, due to the following fact proved in [Til99]

$$
\mathbb{Z} \times B \Gamma_{\infty}^{+} \simeq \Omega^{\infty} \Sigma^{\infty}\left(S^{0}\right)_{\frac{1}{2}} \times W
$$

for some infinite loop space $W$.

Let us conclude this paper by a couple of questions.

Question 2. Find a more general and practically applicable condition on a map of simplicial spaces

$$
p_{*}: X_{*} \longrightarrow Y_{*}
$$

under which $p_{*}$ induces a quasifibration after taking the geometric realization functor. It is also interesting if we could find a condition under which $p_{*}$ induces a Serre fibration.

This question was discussed in a mailing list of algebraic topologists maintained by Don Davis. The question is

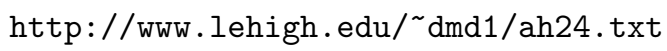

And a response is

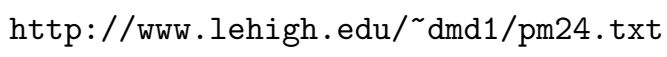

Question 3. Find a nice categorification of Segal's $K$-homology.

As we have seen, Segal's $K$-homology $k u(X ; H)$ is derived from the infinite symmetric product $\mathrm{SP}^{\infty}(X)$ by replacing the "coefficients" $n_{i} \in \mathbb{N}$ of elements

$$
n_{1} x_{1}+\cdots+n_{j} x_{j} \in \mathrm{SP}^{\infty}(X)
$$

by objects of a categorification of $\mathbb{N}$, i.e. the category $\operatorname{Vect}(H)$ of finite dimensional vector spaces in $H$.

From the view point of stable homotopy theory, the (co)homology theory next to $K$-theory is the elliptic (co)homology theory. There is an attempt by Baas, Dundas and Rognes BDR04 to define a form of elliptic cohomology theory by using a categorification of $\operatorname{Vect}(H)$.

They used the category of 2-vector spaces introduced by Kapranov and Voevodsky KV94 and introduces a notion of 2-vector bundle. They succeeded in defining a cohomology theory, although their cohomology theory fails to be complex oriented.

It is natural to try to categorify Segal's $K$-homology to obtain a form of elliptic homology. Note that the definition of Segal's $K$-homology involves with metric. We would need a categorification of the category of vector spaces with inner product. 
Question 4. Explore the properties of the homology theory defined by MadsenTillmann.

One of the reasons why their homology theory contains the stable homotopy is that the construction of $M_{q}(X)$ is similar to that of $C(X ; H)$ in the sense that the disjointness condition in $Y(j)_{q}$ is independent of the labelling points in $X$. On the other hand, the orthogonality conditions in Segal's $K$ homology depends on the labelling points in $X$.

There might be a modification of Madsen-Tillmann construction which is directly related to $K$-theory or elliptic (co)homology. Note that a conformal field theory is a functor from a certain cobordism category to the category of vector spaces.

\section{References}

And78. D. W. Anderson. Fibrations and geometric realizations. Bull. Amer. Math. Soc., 84(5):765-788, 1978.

BDR04. Nils A. Baas, Bjørn Ian Dundas, and John Rognes. Two-vector bundles and forms of elliptic cohomology. In Topology, geometry and quantum field theory, volume 308 of London Math. Soc. Lecture Note Ser., pages 18-45. Cambridge Univ. Press, Cambridge, 2004, arXiv:math.AT/0306027

DT58. Albrecht Dold and René Thom. Quasifaserungen und unendliche symmetrische Produkte. Ann. of Math. (2), 67:239-281, 1958.

Goo03. Thomas G. Goodwillie. Calculus. III. Taylor series. Geom. Topol., 7:645711 (electronic), 2003.

KV94. M. M. Kapranov and V. A. Voevodsky. 2-categories and Zamolodchikov tetrahedra equations. In Algebraic groups and their generalizations: quantum and infinite-dimensional methods (University Park, PA, 1991), volume 56 of Proc. Sympos. Pure Math., pages 177-259. Amer. Math. Soc., Providence, RI, 1994.

May72. J. P. May. The geometry of iterated loop spaces. Springer-Verlag, Berlin, 1972.

May75. J. Peter May. Classifying spaces and fibrations. Mem. Amer. Math. Soc., 1(1, 155):xiii 98, 1975 .

Mil67. R. James Milgram. The bar construction and abelian $H$-spaces. Illinois J. Math., 11:242-250, 1967.

MT01. Ib Madsen and Ulrike Tillmann. The stable mapping class group and $Q\left(\mathbb{C P}_{)}^{\infty}\right.$. Invent. Math., 145(3):509-544, 2001.

Seg73. Graeme Segal. Configuration-spaces and iterated loop-spaces. Invent. Math., 21:213-221, 1973.

Seg74. Graeme Segal. Categories and cohomology theories. Topology, 13:293-312, 1974.

Seg77. Graeme Segal. $K$-homology theory and algebraic $K$-theory. In $K$-theory and operator algebras (Proc. Conf., Univ. Georgia, Athens, Ga., 1975), pages 113-127. Lecture Notes in Math., Vol. 575. Springer, Berlin, 1977.

Shi01. Kazuhisa Shimakawa. Configuration spaces with partially summable labels and homology theories. Math. J. Okayama Univ., 43:43-72, 2001. 
Shi07. Kazuhisa Shimakawa. Labeled configuration spaces and group completions. Forum Math., 19(2):353-364, 2007.

Tam. Dai Tamaki. Twisting Segal's $K$-homology theory. this volume.

Til97. Ulrike Tillmann. On the homotopy of the stable mapping class group. Invent. Math., 130(2):257-275, 1997.

Til99. Ulrike Tillmann. A splitting for the stable mapping class group. Math. Proc. Cambridge Philos. Soc., 127(1):55-65, 1999.

Woo79. Richard Woolfson. Hyper- $\Gamma$-spaces and hyperspectra. Quart. J. Math. Oxford Ser. (2), 30(118):229-255, 1979. 